\title{
High-order spectra-based deconvolution of ultrasonic NDT signals for defect identification
}

\author{
Ahmed Yamani *, Maamar Bettayeb, Lahouari Ghouti \\ King Fahd University of Petroleum and Minerals, KFUPM Box 1811, Dhahran 31261, Saudi Arabia
}

Received 14 April 1997

\begin{abstract}
In ultrasonic nondestructive testing (NDT) of materials, pulse-echo measurements are masked by the characteristics of the measuring instruments, the propagation paths taken by the ultrasonic pulses, and noise. This measured pulse-echo signal is modeled by the convolution of the defect impulse response and the measurement system response, added to noise. The deconvolution operation, therefore, seeks to undo the effect of the convolution and extract the defect impulse response which is essential for defect identification. In this contribution, we show that the defect ultrasonic model can be formulated in the higherorder-spectra (HOS) domain in which the processing is more suitable to unravel the effect of the measurement system and the additive Gaussian noise. In addition, a new technique is developed to faithfully recover the impulse response signal from its HOS. Synthesized ultrasonic signals as well as real signals obtained from artificial defects are used to show that the proposed technique is superior to conventional second-order statistics-based deconvolution techniques commonly used in NDT. (C) 1997 Elsevier Science B.V.
\end{abstract}

Keywords: High-order statistics; High-order spectra; Defect impulse response; Ultrasonic signals deconvolution

\section{Introduction}

Pulse-echo signals measured in ultrasonic NDT include the effect of the measuring systems, the propagation paths taken by the ultrasonic waves, and are corrupted by additive noise. For instance, the ultrasonic signals for a particular reflector recorded under the same conditions, but using different transducers can be quite different. This leads to the difficulty of comparing and analyzing signals, particularly in automated defect identification systems employing different transducers. Generally, it is assumed that the measured pulse-echo is obtained by linearly convolving the defect impulse response with the measured system response. Deconvolution operation therefore seeks to undo the effect of the convolution and extract the defect impulse response which is an essential step for the identification and characterization of defects. Conventional deconvolution techniques such as least square, Wiener filter and minimum variance deconvolution [1] are based on prior knowledge of second-order statistics (SOS) of the noise

\footnotetext{
* Corresponding author.

E-mail: myamani@dpc.kfupm.edu.sa; fax: +96638603535
}

and the input signal. In practice, however, ultrasonic pulse-echo signals are found to be nonminimum phase systems and the acoustic noise due to scattering from the grains inside the propagation medium does not have a readily known statistic [2]. SOS-based deconvolution techniques, being phase-blind, cannot therefore accurately estimate the defect impulse response.

An important criterion for the deconvolution of nonminimum phase systems with non-Gaussian noise statistics is the inclusion of the additional information contained in the higher-order statistics of the signals. The objective of this paper is to formulate the defect ultrasonic model in the polyspectra domain in which the processing is more suitable to unravel the effect of the measurement system and the additive Gaussian noise. In addition, a new technique is developed to faithfully recover the impulse response signal from its polyspectrum. Synthesized as well as real data obtained from artificial defects are used in this paper. Comparison of the impulse response obtained from the proposed HOS-based deconvolution technique and that obtained from its counterpart conventional SOS-based deconvolution technique demonstrates clearly the superiority of the former (proposed) over the later technique. In the 
next section, we present the SOS-based deconvolution scheme, and in Section 3 how this scheme can be extended to higher-order statistics. In Section 4 we develop a new scheme to recover the signal from its bispectrum. Simulation results are presented in Section 5 where it is demonstrated that the HOS-based deconvolution technique is a good candidate to unravel the effects of the measurement system and that the resulting impulse response, being highly accurate, can be used efficiently in any automated defect identifier.

\section{Background}

A measured ultrasonic flaw signal, $y(t)$, can be modeled as the convolution of the measurement system impulse response function, $x(t)$, with the flaw's impulse response function, $h(t)$, plus noise, $N(t)$. The model can be written as

$y(t)=x(t) \otimes h(t)+N(t)$,

where $\otimes$ denotes the convolution operation. With this model, a defect of a particular geometry would be completely characterized by its impulse response. Estimation of $h(t)$ in Eq. (1), given the measured flaw signal and the measurement system impulse response is variously known as system identification, filtering, or simply as deconvolution. Many deconvolution techniques have been developed in different engineering areas ranging from seismic exploration to medical imaging. Chen et al. [1] have studied the feasible application of conventional deconvolution techniques to ultrasonic NDT, and concluded that the Wiener filter is a good candidate for such a purpose.

\subsection{Wiener filtering}

A least-square error estimate of $h(t)$ from Eq. (1) via the frequency domain is given by [3]

$\hat{H}(w)=\frac{Y(w) \cdot X^{*}(w)}{|X(w)|^{2}+S_{n}(w) / S_{h}(w)}$,

where $X(w), Y(w)$ and $\hat{I}(w)$ are the Fourier transforms of $x(t), y(t)$ and $\hat{h}(t)$, respectively, whereas $S_{n}(w)$ and $S_{h}(w)$ are the power spectral densities of $N(t)$ and $h(t)$, respectively. It can be seen from Eq. (2) that the application of 'optimal' Wiener filter requires estimates of the parameters (mean and variance) which describe the noise and scattering amplitude distribution of the defect, respectively. In practice, estimating the parameters which describe the scattering amplitude distribution of the defect is possible only in rare instances. Conversely, reliable estimates of the noise distribution parameters are generally not known. A 'near-optimal' implementa- tion of Wiener filter can be achieved by replacing the ratio of the spectral densities in Eq. (2) by a positive value $q$ commonly defined by

$q=0.01|X(w)|_{\max }^{2}$.

This constant is sometimes known as the 'noisedesensitizing factor'. However, in moderate to low signal-to-noise ratio (SNR) applications, it is clearly unacceptable to achieve the deconvolution via the Wiener filter with the desensitization term taken to be a frequency independent constant which is insensitive to the noise level. Neal et al. [4] have shown that an optimal filter can be achieved that utilizes prior noise information only, with no prior information about the flaw distribution. In practice, however, it is found that ultrasonic pulse-ccho signals are nonminimum phase systems, and that the acoustic noise due to scattering from the grains inside the material is not neccssarily Gaussian. Thus, optimal implementation (even if possible) of the Wiener filter does not necessarily garantee accurate estimation of the defect impulse response. This drawback is alleviated when higher-order statistics-based deconvolution (via cumulants) of the pulse-echo are used.

\section{Higher-order statistics and spectra}

In this section, we first introduce the definitions, and the properties of higher-order statistics, i.e. moments and cumulants, and their corresponding higher-order spectra. Emphasis is placed on the second-, third- and fourth-order statistics and their respective Fourier transforms: power spectrum, bispectrum and trispectrum.

If $\{X(k)\}, k=0, \pm 1, \pm 2, \pm 3, \ldots$ is a real stationary discrete-time signal and its moments up to order $n$ exist, then

$m_{n}^{x}\left(\tau_{1}, \tau_{2}, \ldots, \tau_{n}\right)=E\left\{X(k) X\left(k+\tau_{1}\right) X\left(k+\tau_{2}\right) \ldots X\left(k+\tau_{n}\right)\right\}$,

represents the $n$ th-order moment function of the signal, and $E\{$.$\} denotes statistical expectation. Clearly, the$ second-order moment function, $m_{2}^{x}\left(\tau_{1}\right)$, is the autocorrelation of $\{X(k)\}$.

The $n$ th-order cumulant function of a non-Gaussian stationary random signal $X(k)$ can be written as (for $n=3,4$ only):

$$
\begin{aligned}
c_{n}^{x}\left(\tau_{1}, \tau_{2}, \ldots, \tau_{n-1}\right)= & m_{n}^{x}\left(\tau_{1}, \tau_{2}, \ldots, \tau_{n-1}\right) \\
& -m_{n}^{G}\left(\tau_{1}, \tau_{2}, \ldots, \tau_{n-1}\right),
\end{aligned}
$$

where $m_{n}^{\mathrm{G}}\left(\tau_{1}, \tau_{2}, \ldots, \tau_{n-1}\right)$ is the $n$ th-order moment function of an equivalent Gaussian signal that has the same mean value and autocorrelation sequence as $X(k)$. 
Clearly, the $n$ th-order $(n>2)$ cumulant of a Gaussian process is zero.

Higher-order spectra are the multi-dimensional Fourier transforms of higher-order statistics. Thus, the power spectrum, bispectrum and trispectrum are the one-, two- and three-dimensional Fourier transforms of the cumulants of order 2, 3 and 4, respectively. For instance, the bispectrum of the signal $X(k)$ is defined as

$$
\begin{aligned}
C_{3}^{x}\left(w_{1}, w_{2}\right)= & \sum_{\tau_{1}=-\infty}^{+\infty} \sum_{\tau_{2}=-\infty}^{+\infty} c_{3}^{x}\left(\tau_{1}, \tau_{2}\right) \\
& \times \exp \left[-j\left(w_{1} \tau_{1}+w_{2} \tau_{2}\right)\right],
\end{aligned}
$$

whereas the trispectrum is defined as

$$
\begin{aligned}
C_{4}^{x}\left(w_{1}, w_{2}, w_{3}\right)= & \sum_{\tau_{1}=-\infty}^{+\infty} \sum_{\tau_{2}=-\infty}^{+\infty} \sum_{\tau_{3}=-\infty}^{+\infty} c_{4}^{x}\left(\tau_{1}, \tau_{2}, \tau_{4}\right) \\
& \times \exp \left[-j\left(w_{1} \tau_{1}+w_{2} \tau_{2}+w_{3} \tau_{3}\right)\right],
\end{aligned}
$$

Cumulant spectra are more useful in the processing of random signals than are moment spectra becausc

- cumulant spectra of order $n>2$ are zero if the signal is Gaussian, and thus nonzero cumulants spectra provide a measure of extent of non-Gaussianity;

- the cumulants of (higher-order) white noise are multidimentional impulse functions, and the polyspectra of this noise are multidimensionally flat;

- the cumulant of the sum of two statistically independent random processes is equal to the sum of the cumulants of the individual random processes, whereas the same is not true for higher-order moments;

- the bispectrum of a symmetrically distributed random process is zero.

Using the HOS properties Nikias et al. [3], Eq. (1) can be written in HOS domain as

$$
\begin{aligned}
C_{n}^{y}\left(w_{1}, w_{2}, \ldots, w_{n-1}\right)= & C_{n}^{x}\left(w_{1}, w_{2}, \ldots, w_{n-1}\right) H\left(w_{1}\right) \\
& \times H\left(w_{2}\right) \ldots H^{*}\left(w_{1}+w_{2}+\ldots+w_{n-1}\right) \\
& +C_{n}^{N}\left(w_{1}, w_{2}, \ldots, w_{n-1}\right),
\end{aligned}
$$

where $H(w)$ is the Fourier transform of the defect impulse response $h(t)$. In a similar fashion, Eq. (8) can be expressed as

$$
\begin{aligned}
C_{n}^{y}\left(w_{1}, w_{2}, \ldots, w_{n-1}\right)= & C_{n}^{x}\left(w_{1}, w_{2}, \ldots, w_{n-1}\right) \\
& \times C_{n}^{h}\left(w_{1}, w_{2}, \ldots, w_{n-1}\right) \\
& +C_{n}^{N}\left(w_{1}, w_{2}, \ldots, w_{n-1}\right)
\end{aligned}
$$

where $C_{n}^{h}\left(w_{1}, w_{2}, \ldots, w_{n-1}\right)$ is the polyspectrum of $h(t)$. In view of the attractive properties of higher-order spectra, the computation complexity of the polyspectra as the order is increased, and the simplicity to formulate the ultrasonic defect model, only the bispectrum is used in this work. Eq. (1) is rewritten in terms of the bi- spectrum as

$$
C_{3}^{y}\left(w_{1}, w_{2}\right)=C_{3}^{x}\left(w_{1}, w_{2}\right) C_{3}^{h}\left(w_{1}, w_{2}\right) \text {. }
$$

It is clear from Eq. (10) that the noise does not have to be Gaussian to be filtered out, but it can have any symmetrical probability density function (PDF) (such as Laplace, uniform, Bernouli-Gaussian distributions). Thereafter, the noise-free bispectrum of the defect impulse response is used to recover $h(t)$ using a new recovery method that will be described in the next section.

\section{Signal recovery from its bispectrum}

We have seen in the previous section that the bispectrum of the defect impulse response can easily be computed from Eq. (10). Any additive white noise with a symmetrical PDF is automatically filtered out, and the objective therefore, is to recover the signal from its bispectrum. This operation is not a one-to-one transformation, but it requires considerable efforts [5-7] and a prior phase information (usually not available) to achieve this goal. Moreover, the recovered signal is contaminated with an estimation error that can have, in some cases, a high variance. We have developed in this paper a simple but accurate technique capable of recovering the signal from its bispectrum by using the relationship between the bicepstra and the bispectra defined by Pan and Nikias [8] as

$m_{1} b_{3}^{h}\left(m_{1}, m_{2}\right)=F_{2}^{-1}\left[\frac{F_{2}\left[\tau_{1} \cdot c_{3}^{h}\left(\tau_{1}, \tau_{2}\right)\right]}{F_{2}\left[c_{3}^{h}\left(\tau_{1}, \tau_{2}\right)\right]}\right]$,

where $F_{2}^{-1}[\cdot]$ denotes the two-dimensional inverse Fourier transform, $b_{3}^{h}(\cdot)$ is the third-order cepstrum (or bi-cepstrum) of the impulse response $h(t)$, and $m_{1}$ is the variable along axis 1 in the bicepstrum domain. Using the properties of the Fourier transform, Eq. (11) can be written as

$m_{1} b_{3}^{h}\left(m_{1}, m_{2}\right)=F_{2}^{-1}\left\{\frac{j \frac{\partial}{\partial w_{1}}\left[C_{3}^{h}\left(w_{1}, w_{2}\right)\right]}{C_{3}^{h}\left(w_{1}, w_{2}\right)}\right\}$

where $\partial / \partial w_{1}(\cdot)$ is the partial derivative with respect to $w_{1}$, and $j=\sqrt{-1}$.

Eq. (12) is the basis of the recovery technique. First, it shows an alternative relationship between the bicepstrum and the bispectrum using the derivative of the logarithm rather than the logarithm relation traditionally used. Second, it does not require any phase unwrapping required by the logarithmic relation. This enables the recovery method to be highly accurate when compared to other recovery techniques [5-7]. For linear 
systems, it is known that the bicepstrum is completely defined along the three lines given by $m_{1}=0, m_{2}=0$, and $m_{1}=m_{2}$ as

$$
b_{3}^{h}\left(m_{1}, m_{2}\right)= \begin{cases}\alpha, & m_{1}=0, m_{2}=0, \\ -A^{\left(m_{2}\right)} / m_{2}, & m_{1}=0, m_{2}>0, \\ -A^{\left(m_{1}\right)} / m_{1}, & m_{1}>0, m_{2}=0, \\ B^{\left(-m_{1}\right)} / m_{1}, & m_{1}<0, m_{2}=0, \\ -B^{\left(-m_{2}\right)} / m_{2}, & m_{1}=0, m_{2}<0, \\ -A^{\left(m_{2}\right)} / m_{2}, & m_{1}=m_{2}>0, \\ B^{\left(-m_{2}\right)} / m_{2}, & m_{1}=m_{2}<0, \\ 0, & \text { otherwise. }\end{cases}
$$

and a simple procedure for the computation of the impulse response $h(t)$ using the bicepstral parameters $A^{(m)}$ and $B^{(m)}$ [9] can be used. The proposed algorithm used to extract the defect impulse response, $h(t)$, from the knowledge of $x(t), y(t)$ using the proposed HOSbased deconvolution method is as follows:

(1) Given the system response, $x(t)$, and the defect echo, $y(t)$, calculate their bispectrum.

(2) For an additive white noise with a symmetrically distributed PDF, obtain the bispectrum of the defect from Eq. (10).

(3) Calculate the bicepstrum of the defect using Eq. (12).

(4) Obtain the bicepstral parameters from Eq. (13).

(5) Use the Oppenheim and Schafer algorithm [9] to compute $h(t)$ from the knowledge of the bicepstral parameters.

\section{Simulation and results}

In this section, we first use synthesized data generated using the model shown in Fig. 1 to test and compare the proposed deconvolution technique with the Wiener filter. Thereafter, real ultrasonic data obtained from artificial defects is used.

The input signal, $x(t)$, is taken as a Gaussian pulse that is amplitude modulating a single tone carrier whose frequency lies in the ultrasonic range. The noise, $n(t)$, having a normally distributed PDF, is scaled by a factor to account for the different SNR used. The impulse response, $h(t)$, is modeled by the following system which is known to have a pronounced maximum phase part, and having a transfer function given by [3]

$H(z)=0.2197 z-0.747+0.6085 z^{-1}+0.1533 z^{-2}$.

Estimation of the impulse response $h(t)$ from the knowledge of the $(x(t), y(t))$ pair only is carried out using Wiener filter and our proposed technique. Fig. 2 shows $x(t)$

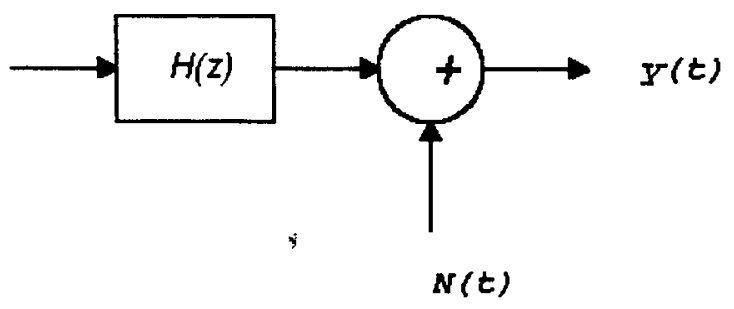

Fig. 1. Ultrasonic defect model.

this estimated impulse response at a SNR of $40 \mathrm{~dB}$. For comparison, the true impulse response is also displayed. For such a high SNR, the Wiener filter is estimating an acceptable impulse response as expected. However, for lower SNR, this estimated impulse response deviates considerably from the true one, while the bispectrumbased method produces a highly accurate estimate. Fig. 3 illustrates this result for a SNR of $10 \mathrm{~dB}$.

Next, the performance of the proposed technique is tested at different SNR. An estimation error variance is computed to quantify this performance. Fig. 4 shows the variation of this estimation error variance with SNR. As expected, the HOS-based deconvolution technique produces accurate estimate of the impulse response even at extremely low SNR. For comparison, the performance of the Wiener filter is also included, where it can be seen that the proposed technique outperforms its counterpart SOS-based deconvolution technique. For instance, the performance of the proposed technique at $-5 \mathrm{~dB}$ SNR is equivalent to the performance of Wiener filter when the SNR is more than $70 \mathrm{~dB}$. This can be attributed to the inherent high-frequency oscillations in the impulse response estimated [1] using SOS-based deconvolution techniques. This oscillation is also seen in the estimated impulse response using Wiener filter which is shown in Figs. 2 and 3, while the HOS-based
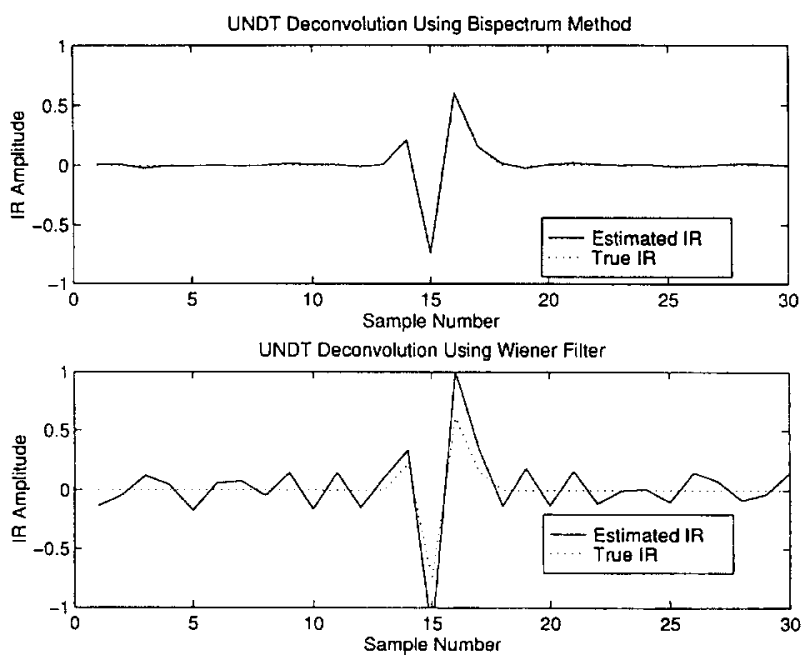

Fig. 2. Impulse response computed using SOS- and HOS-based deconvolution with $\mathrm{SNR}=40 \mathrm{~dB}$. 

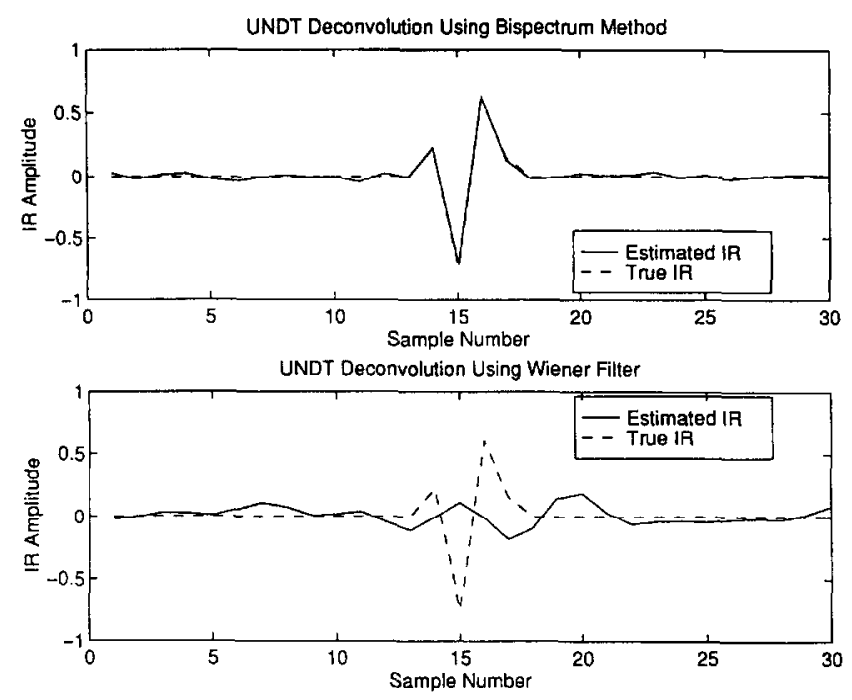

Fig. 3. Impulse response computed using SOS- and HOS-based deconvolution. with $\mathrm{SNR}=10 \mathrm{~dB}$.

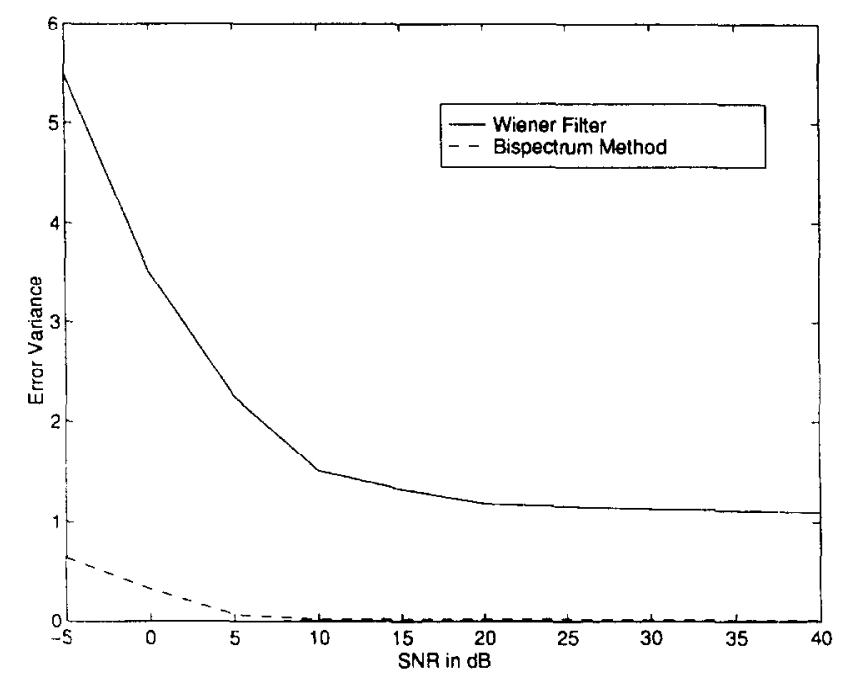

Fig. 4. The variance of the estimation error versus SNR.

deconvolution, being highly accurate even at extremely low SNR, produces oscillation-free impulse response.

The proposed deconvolution technique is tested using real data [10], which are part of a larger data set obtained from the Army's Material Technology laboratory (Watertown, MA). The ultrasonic signals used are shown in Fig. 5, namely T15A0, T15A1 and T15A2. $\mathrm{T} 15 \mathrm{~A} 0$ is the measurement system impulse response, also known as the reference signal, while T15A1 and T15A2 are, respectively, the pulse-echoes from a flatcut hole (A1) and an angular-cut holc (A2) with equal diameters of $32 \mathrm{~mm}$ (see Ref. [10] for an illustration of these defect geometries). The center frequency of the transducer used is $15 \mathrm{MHz}$, and the A-scan signals contain 512 data points digitized at a rate of $100 \mathrm{MHz}$. The impulse responses of these artificial defects estimated using the HOS-based deconvolution technique are shown in Figs. 6 and 7. As expected from the
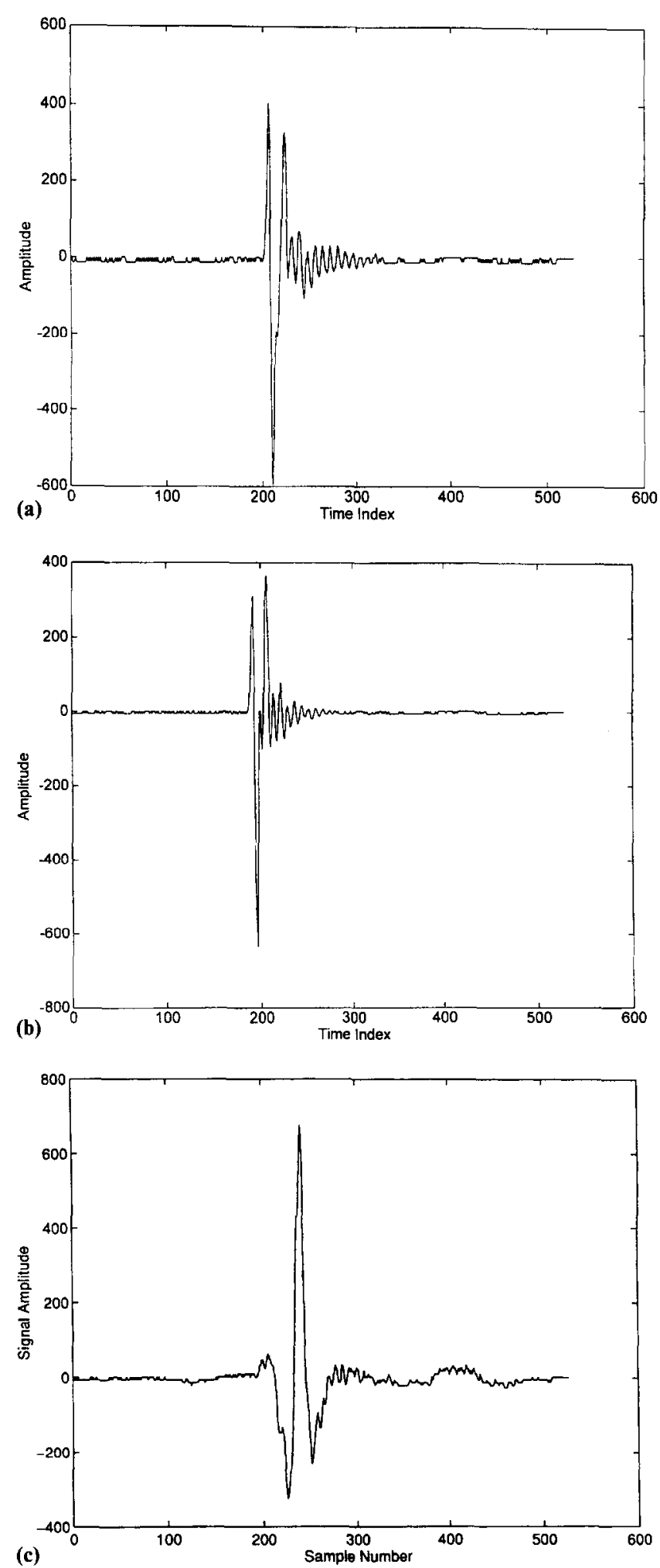

Fig. 5. Real ultrasonic signals used. (a) Flawless sample signal T15A0; (b) flat-cut hole signal T15AI; (c) angular-cut hole signal T15A2.

simulation results, these impulse responses are oscillation-free. In addition, as the ultrasonic signals used have a high SNR, the resulting defect impulse responses should be very close to the 'true' ones. 


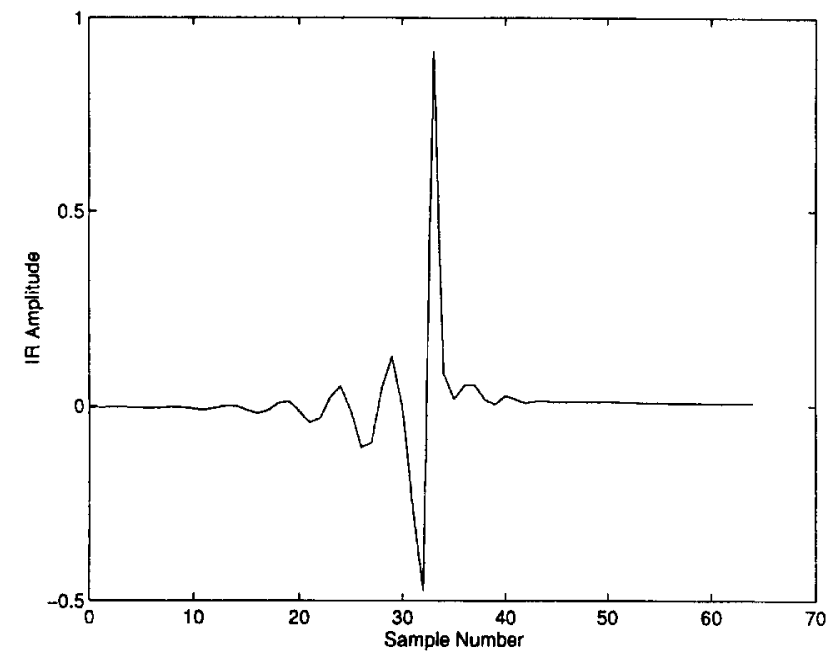

Fig. 6. Impulse response of defect Al (flat-cut hole) obtained using HOS-based deconvolution.

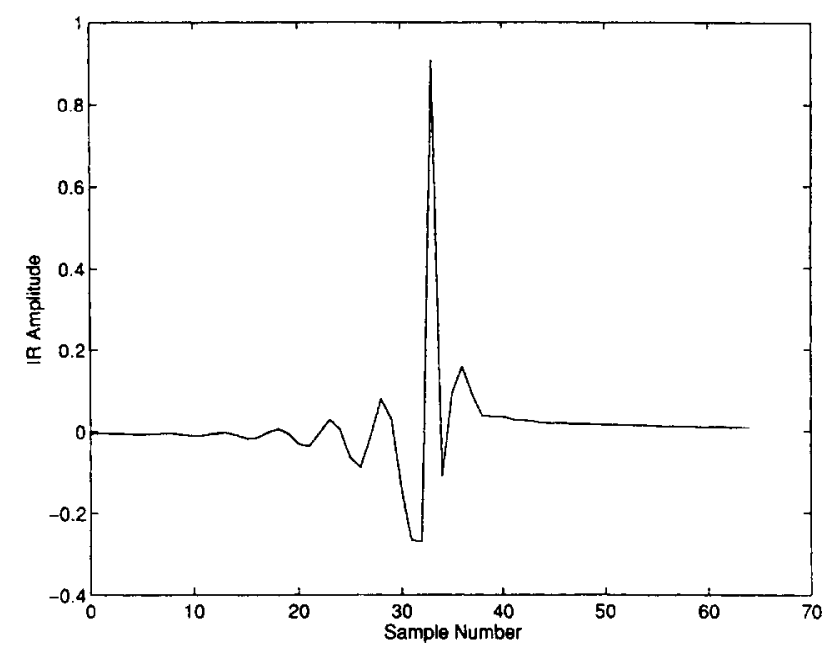

Fig. 7. Impulse response of defect A2 (angular-cut hole) obtained using HOS-based deconvolution.

\section{Conclusion and discussions}

The defect ultrasonic model described by a convolutional operation is adopted in many NDT problems. Extraction of a signal that is insensitive to the noise and the measurement system, but dependent on the defect type only, is the ultimate goal and the challenge for today's NDT scicntific and engineering communities. As ultrasonic pulse-echoes are found to be nonminimum phase systems, and that the acoustic noise due to scattering from the grains inside the propagation media is not necessarily Gaussian, SOS-based deconvolution techniques cannot therefore accurately estimate the defect impulse response, as they are phase blind.

In this contribution, we have formulated the defect ultrasonic model in the higher-order spectrum domain in which the processing is more suitable to easily filter out any additive noise with a symmetrically distributed PDF. Recovering the defect impulse response signal from its bispectrum is not a one-to-one operation, but it requires considerable efforts and a prior phase information to achieve this goal. In addition, the recovered signals using existing techniques are usually contaminated by high variance estimation errors. We have developed in this work a simple but accurate recovery technique that does not require any phase unwrapping, or any prior information to be used. Simulation results using synthesized data demonstrate that the proposed deconvolution technique is a good candidate that is capable of faithfully recovering the defect impulse response from signals with extremely low SNR. Real ultrasonic signals are used to extract impulse responses of artificial defects, which are tested and found to be nonminimum phase systems. Moreover, it can be noticed that unlike the SOS-based deconvolution techniques [1] which produce high frequency oscillations in the defect impulse response, the proposed technique, being highly accurate even at extremely low SNR, has yielded oscillation-free defect impulse responses. Future work will focus on the use of this HOS-based deconvolution technique on real defect signals using different transducers.

\section{Acknowledgement}

This work is supported in part through research grant provided by SABIC under the grant EE/SABIC/96-8. The authors would like to express their thanks to Professor C.H. Chen of the University of Massachusetts for his valuable help and comments.

\section{References}

[1] C.H. Chen, S.K. Sin, High resolution deconvolution techniques and their applications in ultrasonic NDE, International Journal of Image Systems and Techniques 1 (1989) 223.

[2] S.P. Neal, K.D. Donohue, Testing for non Gaussian fluctuations in grain noise, QNDE 15 (1996) 1.

[3] C.L. Nikias, A.P. Petropulu, Higher-Order Spectra Analysis: A Nonlinear Signal Processing Framework, Prentice Hall, Englewood Cliffs, NJ, 1993.

[4] S.P. Neal, P.L. Speckman, M.A. Enright, Flaw signature estimation in ultrasonic nondestructive evaluation using Wiener filter with limited prior information, IEEE Transactions on Ultrasonic Ferroelectrics and Frequency Control 40 (4) (1993) 347.

[5] T. Matsuoka, T.J. Ulrych, Phase estimation using the bispectrum, IEEE Proceedings 72 (10) (1984) 1403.

[6] H. Bartelt, A.W. Lohmann, B. Wirnitzer, Phase and amplitude recovery from bispectrum, Applied Optics 23 (18) (1984) 3121.

[7] D.R. Brillinger, M. Rosenblatt, Asymptotic theory of $k$ th-order spectra, in: B. IIarris (Ed.), Spectral Analysis of Time Series, Wiley, New York, 1967, Chapter 3, p. 153. 
[8] R. Pan, C.L. Nikias, The complex spectrum of higher-order cumulants and non minimum phase system identification, IEEE Transactions on ASSP 36 (2) (1988) 185.

[9] A.V. Oppenheim, R.W. Schafer, Discrete-time Signal Processing, Prentice Hall. Englewood Cliffs, NJ, 1989.
[10] C.H. Chen, High resolution deconvolution techniques and their applications in ultrasonic NDE, International Journal of Imaging Systems and Techniques 1 (1989) 223. 